Publ. RIMS, Kyoto Univ.

40 (2004), 991-1014

\title{
The Uniformity Principle on Traced Monoidal Categories ${ }^{\dagger}$
}

By

Masahito HASEGAWA*

\begin{abstract}
The uniformity principle for traced monoidal categories has been introduced as a natural generalization of the uniformity principle (Plotkin's principle) for fixpoint operators in domain theory. We show that this notion can be used for constructing new traced monoidal categories from known ones. Some classical examples like the Scott induction principle are shown to be instances of these constructions. We also characterize some specific cases of our constructions as suitable enriched limits.
\end{abstract}

\section{§1. Introduction}

Traced monoidal categories were introduced by Joyal, Street and Verity [16] as the categorical structure for cyclic phenomena arising from various areas of mathematics, most notably knot theory [25]. They are (balanced) monoidal categories [15] enriched with a trace, which is a natural generalization of the traditional notion of trace in linear algebra, and can be regarded as an operator to create a "loop". For example, the braid closure operation - e.g. the construction of the trefoil knot from a braid depicted below - can be considered as the trace operator on the category of tangles.

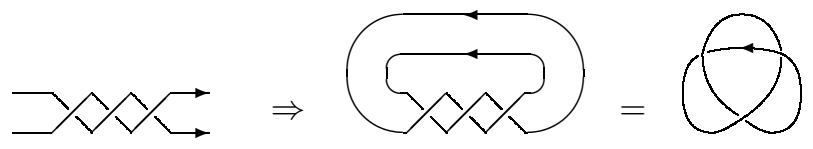

Communicated by R. Nakajima. Received December 16, 2003.

2000 Mathematics Subject Classification(s): 18D10, 19D23

${ }^{\dagger}$ This article is an invited contribution to a special issue of Publications of RIMS commemorating the fortieth anniversary of the founding of the Research Institute for Mathematical Sciences.

* Research Institute for Mathematical Sciences, Kyoto University, Kyoto 606-8502, Japan; PRESTO, Japan Science and Technology Agency, Japan 
In computer science, specialized versions of traced monoidal categories naturally arise from considerations on recursion/feedback operators (in the fairly general sense) as well as cyclic data structures. Here is a graphical account of the recursive (fixpoint) computation created from a circularly shared structure:

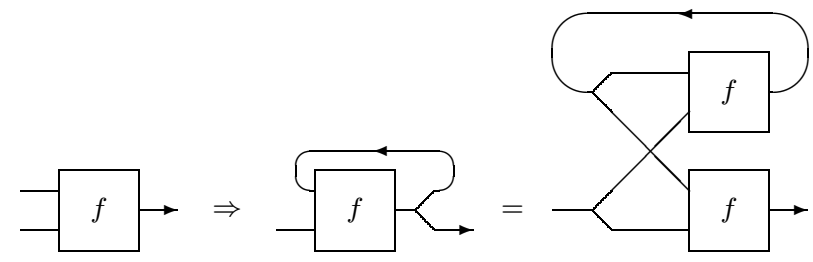

In the middle of 90's, Martin Hyland and the author independently observed a bijective correspondence between Conway (Bekič, or dinatural diagonal) fixpoint operators $[3,23]$ and traces on categories with finite products $[8,9]$. Thus, in this setting, the notion of trace precisely captures the well-behaved fixpoint operators commonly used in computer science.

An extra bonus of this trace-fixpoint correspondence is that the uniformity principle (à la Plotkin) on a fixpoint operator [20, 6, 23] precisely amounts to a uniformity principle on the corresponding trace, as introduced in the previous work $[8,9]$ (see also historical remarks at the end of Sec. 3). This uniformity principle is general enough to make sense for arbitrary traced monoidal categories. An application of this concept is found in Selinger's work on categorical models of asynchronous communications [21].

In the present paper we demonstrate that this uniformity principle on traced monoidal categories can be used for constructing new traced monoidal categories (and categories with fixpoint operators) from known ones. The construction is very simple and in some sense old - its origin can be traced back to the Scott induction principle.

We must admit that, by its general nature, our constructions do not immediately provide mathematically strong results. However, we think that our development is a natural one, in that it adds a new view to the relationship between model construction techniques and proof principles; in particular, we expect that results of this paper can be combined with the well-established relationship between categorical glueing and logical relations.

Moreover, our constructions seem to enjoy characterizations by some universal property, as certain limits in an enriched sense. We study this issue for some specific cases, point out the difficulties in this direction, and propose a solution for which our leading example (with a slight modification) is characterized as a Graph-enriched limit. 
The rest of this paper is organized as follows. In Sec. 2, we explain the graphical notations for symmetric monoidal categories which are used throughout this paper, and recall the notion of traced symmetric monoidal categories. We then introduce the notion of uniformity and strict maps for traced monoidal categories in Sec. 3. Sec. 4 is devoted for some basic results on the uniformity principle. In Sec. 5 we recall the correspondence between traces and fixpoint operators and give the relationship between the uniformity principle for traces and that for Conway operators. Sec. 6 and 7 form the central part of this paper, where we show how the uniformity principle can be used for constructing new traced monoidal categories. Sec. 8 gives some observations on the uniformity principle and the Int construction.

This paper is a revised and extended version of the work presented at the Category Theory and Computer Science (CTCS'02) conference [10].

Remark 1.1. Although many of the notions and results in this work apply to general traced balanced monoidal categories (where we may have nontrivial intertwiners and twists $[15,22]$ which are essential for interpreting the ribbon diagrams [25]), in this paper we restrict our attention only to traced symmetric monoidal categories, firstly because for ease of presentation, and secondly because most of examples relevant to computer science seem to be instances of symmetric monoidal categories. So by a traced monoidal category, in this paper we mean a traced symmetric monoidal category unless otherwise stated.

\section{§. Preliminaries}

\section{$\S 2.1$ Graphic presentation of monoidal categories}

In this paper, an arrow $f: A_{1} \otimes A_{2} \otimes \cdots \otimes A_{m} \rightarrow B_{1} \otimes B_{2} \otimes \cdots \otimes B_{n}$ in a monoidal category $[19,15]$ (pretended as if the tensor product is strictly associative for brevity) is drawn as (from left to right)

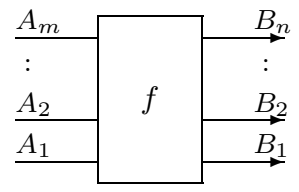

The identity morphism is drawn just as a straight arrow. The composition of $f: X \rightarrow Y$ and $g: Y \rightarrow Z$ is represented as a sequential composition 


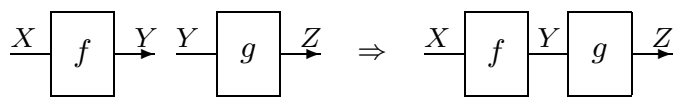

while the tensor $f \otimes g: A \otimes C \rightarrow B \otimes D$ of $f: A \rightarrow B$ and $g: C \rightarrow D$ is drawn as a parallel composition

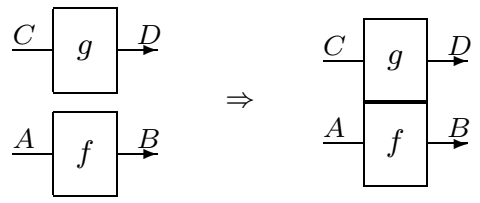

The symmetry $c_{X, Y}: X \otimes Y \rightarrow Y \otimes X$ in a symmetric monoidal category is represented by a cross wiring:

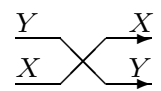

A formal justification of these graphical presentations has been given by Joyal and Street [14].

\section{§2.2. Traced monoidal categories}

A trace on a symmetric monoidal category $\mathcal{C}$ is a family of functions $\operatorname{Tr}_{A, B}^{X}$ : $\mathcal{C}(A \otimes X, B \otimes X) \rightarrow \mathcal{C}(A, B)$ subject to the following conditions:

- it is natural in $A$ and $B$ (left/right tightening), and dinatural in $X$ (sliding)

- vanishing: $\operatorname{Tr}_{A, B}^{I}(f)=f$ and $\operatorname{Tr}_{A, B}^{X \otimes Y}(f)=\operatorname{Tr}_{A, B}^{X}\left(\operatorname{Tr}_{A \otimes X, B \otimes X}^{Y}(f)\right)$

- superposing: $\operatorname{Tr}_{C \otimes A, C \otimes B}^{X}\left(i d_{C} \otimes f\right)=i d_{C} \otimes \operatorname{Tr}_{A, B}^{X}(f)$

- yanking: $\operatorname{Tr}_{X, X}^{X}\left(c_{X, X}\right)=i d_{X}$

where, again for brevity, we pretend as if the tensor is strictly associative. A traced symmetric monoidal category is a symmetric monoidal category equipped with a (specified) trace - note that there can be many ways of giving traces.

Trace admits a natural graphical presentation as a "feedback": for $f$ : $A \otimes X \rightarrow B \otimes X$, its trace $\operatorname{Tr}_{A, B}^{X}(f): A \rightarrow B$ can be drawn as

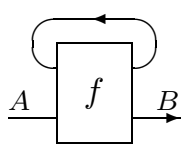

Using this notation, the axioms for trace given above can be graphically presented as follows. 
Naturality (Left Tightening)
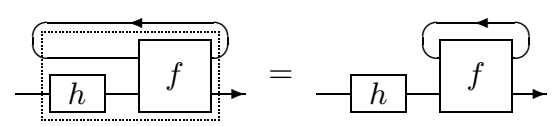

Naturality (Right Tightening)

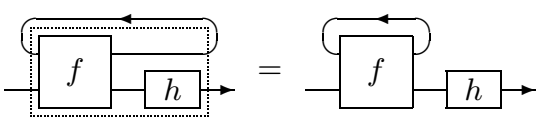

Dinaturality (Sliding)

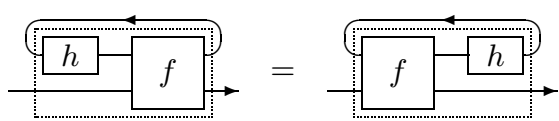

Vanishing $(I)$
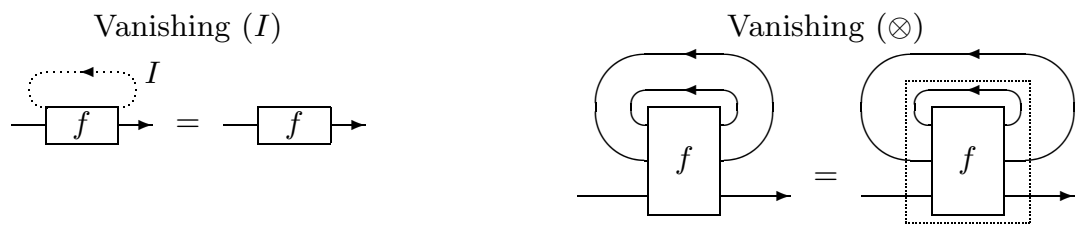

Superposing

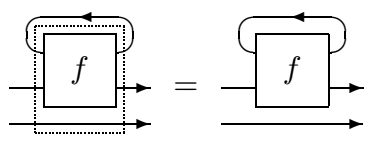

Yanking

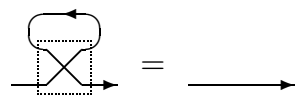

\section{$\S 3 . \quad$ Uniformity for Traces}

Definition 3.1. $\quad$ Consider a traced monoidal category $\mathcal{C}$ with trace $\mathrm{Tr}$. We say $h: X \rightarrow Y$ is strict in $\mathcal{C}$ (with respect to the trace $T r$ ) if the following condition holds:

For any $f: A \otimes X \rightarrow B \otimes X$ and $g: A \otimes Y \rightarrow B \otimes Y$, $\left(i d_{B} \otimes h\right) \circ f=g \circ\left(i d_{A} \otimes h\right): A \otimes X \rightarrow B \otimes Y$

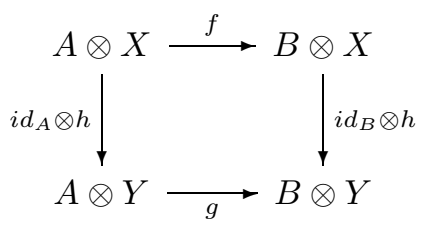

implies $\operatorname{Tr}_{A, B}^{X}(f)=\operatorname{Tr}_{A, B}^{Y}(g): A \rightarrow B$.

In terms of the graphic notation:

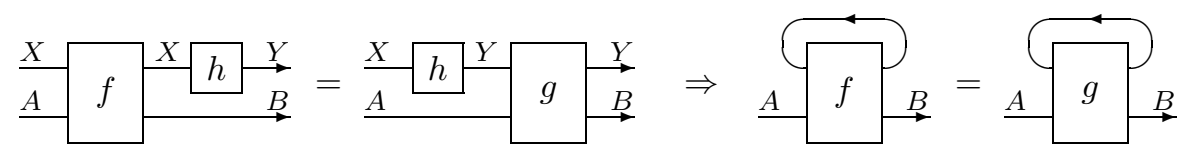


Definition 3.2. $\quad$ Let $\mathcal{C}$ be a traced monoidal category with trace $\mathrm{Tr}$, and $\mathcal{S}$ be a class of arrows of $\mathcal{C}$. We say $\operatorname{Tr}$ is uniform (or: $\operatorname{Tr}$ satisfies the uniformity principle) for $\mathcal{S}$ if, for any $h: X \rightarrow Y$ of $\mathcal{S}$, the condition in Def. 3.1 holds.

Thus the class of strict maps is the largest class of arrows for which the trace satisfies the uniformity principle.

As noted by Selinger [21], the uniformity principle can be seen a proof principle for showing two traces are equivalent: to prove $\operatorname{Tr}^{X}(f)=\operatorname{Tr}^{Y}(g)$, it suffices to find $h: X \rightarrow Y$ of $\mathcal{S}$ such that $(i d \otimes h) \circ f=g \circ(i d \otimes h)$ holds. In such applications, it is often convenient to give $\mathcal{S}$ a priori as a suitable subcategory containing reasonably rich class of arrows; see $i b i d$. for several good examples.

However, we note that there is no reason to expect that the class of strict maps form a category - in fact there are counterexamples, as we will see shortly. For now, we shall recall some popular examples, where strict maps actually form categories.

\section{Example 3.1.}

- The category of finite dimensional vector spaces over a field and linear maps, where the trace is a natural generalization of the standard trace [16]: for a linear map $f: U \otimes_{K} W \rightarrow V \otimes_{K} W$, its trace $\operatorname{Tr}_{U, V}^{W}(f): U \rightarrow V$ is given by

$$
\left(\operatorname{Tr}_{U, V}^{W}(f)\right)_{i, j}=\Sigma_{k} f_{i \otimes k, j \otimes k}
$$

-if $U=V=K$, we have $\operatorname{Tr}^{W}(f)=\Sigma_{k} f_{k, k}$ as expected. An arrow in this category is strict if and only if it is an isomorphism.

- The category Cppo of $\omega$-cpo's with bottom and continuous functions, where the trace is induced from the least fixpoint operator (see Sec. 5). Explicitly, for $f: A \times X \rightarrow B \times X$ we have $\operatorname{Tr}_{A, B}^{X}(f): A \rightarrow B$ by

$$
\operatorname{Tr}_{A, B}^{X}(f)(a)=\pi_{B, X}\left(\operatorname{fix}_{B \times X}\left(\lambda\left(b^{B}, x^{X}\right) \cdot f(a, x)\right)\right)
$$

where fix $_{B \times X}:(B \times X)^{B \times X} \rightarrow B \times X$ is the least fixpoint operator. An arrow is strict w.r.t. this trace iff it preserves the bottom element.

- The category of sets and partial functions, with coproducts as monoidal products - its trace is derived from the natural feedback operator: for 
$f: A+X \rightarrow B+X$ we have $\operatorname{Tr}_{A, B}^{X}(f): A \rightarrow B$ by

$$
\begin{aligned}
& \operatorname{Tr}_{A, B}^{X}\left(f^{A+X \rightarrow B+X}\right)\left(a^{A}\right)= \\
& \quad \text { case } f\left(\operatorname{in}_{A}(a)\right) \text { of }\left(\begin{array}{ccc}
\operatorname{in}_{B}(b) & \mapsto & b \\
\operatorname{in}_{X}(y) & \mapsto & \text { feedback }_{X, B}\left(f \circ \operatorname{in}_{X}\right)(y)
\end{array}\right)
\end{aligned}
$$

where

$$
\text { feedback }_{X, B}\left(g^{X \rightarrow B+X}\right)\left(x^{X}\right)=\text { case } g(x) \text { of }\left(\begin{array}{ccc}
\operatorname{in}_{B}(b) & \mapsto & b \\
\operatorname{in}_{X}(y) & \mapsto & \text { feedback }_{X, B}(g)(y)
\end{array}\right)
$$

In this setting any arrow is strict.

Remark 3.1. Our terminology ("uniformity" and "strictness") is motivated from that of fixpoint operators in domain theory, and will be justified in Sec. 5. The corresponding notions for various specialized versions of traced monoidal categories had appeared in the literature under various names and forms. In particular, Ştefănescu's "enzymatic rule" for his network algebras [24] precisely corresponds to our uniformity principle, where strict arrows are called "functorial arrows" (following the terminology by Arbib and Manes for partially additive categories [2]). See $i b i d$. for bibliographic remarks and also several examples.

\section{$\S 4$. Basic Facts}

As a warming up, let us see a few basic (but fundamental) properties which strict maps in a traced monoidal category enjoy - and do not enjoy. In summary, we will see that (1) isomorphisms are strict, (2) strict maps are closed under tensor product, but (3) strict maps may not be closed under composition. To help with the intuition, we shall extensively make use of the graphical presentation in the proofs below.

Lemma 4.1. In a traced monoidal category, isomorphisms are strict.

Proof. Suppose $h$ is an isomorphism. Then

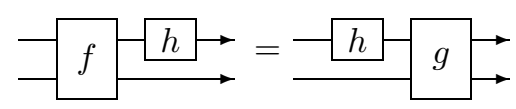


implies

$$
\begin{aligned}
& \underset{f \rightarrow f}{\longrightarrow h} h_{h}^{-1} \quad h^{-1} \circ h=i d \\
& =\underset{h^{-1}}{\stackrel{4}{\longrightarrow}} \stackrel{\longrightarrow}{\longrightarrow} \text { dinaturality } \\
& =\stackrel{h-1}{h} \rightarrow \text { assumption }
\end{aligned}
$$

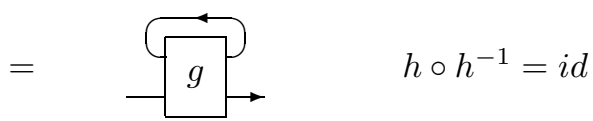

Hence $h$ is strict.

By a similar argument we also have

Lemma 4.2. The composition of a strict map and an isomorphism is strict.

(This actually subsumes the previous lemma, as an identity is trivially strict.)

Lemma 4.3. $\quad$ Strict maps are closed under tensor product.

Proof. Suppose $h$ and $k$ are strict. Then

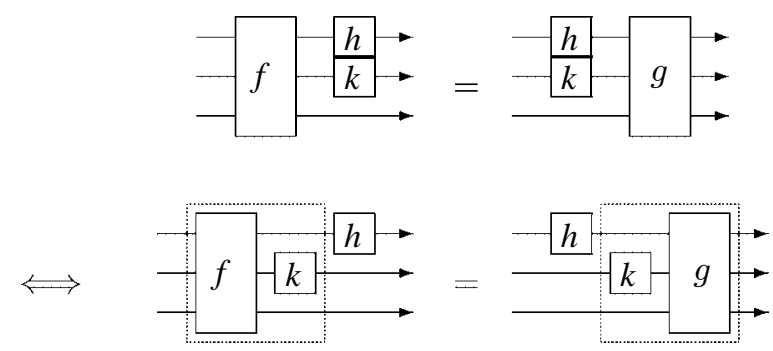




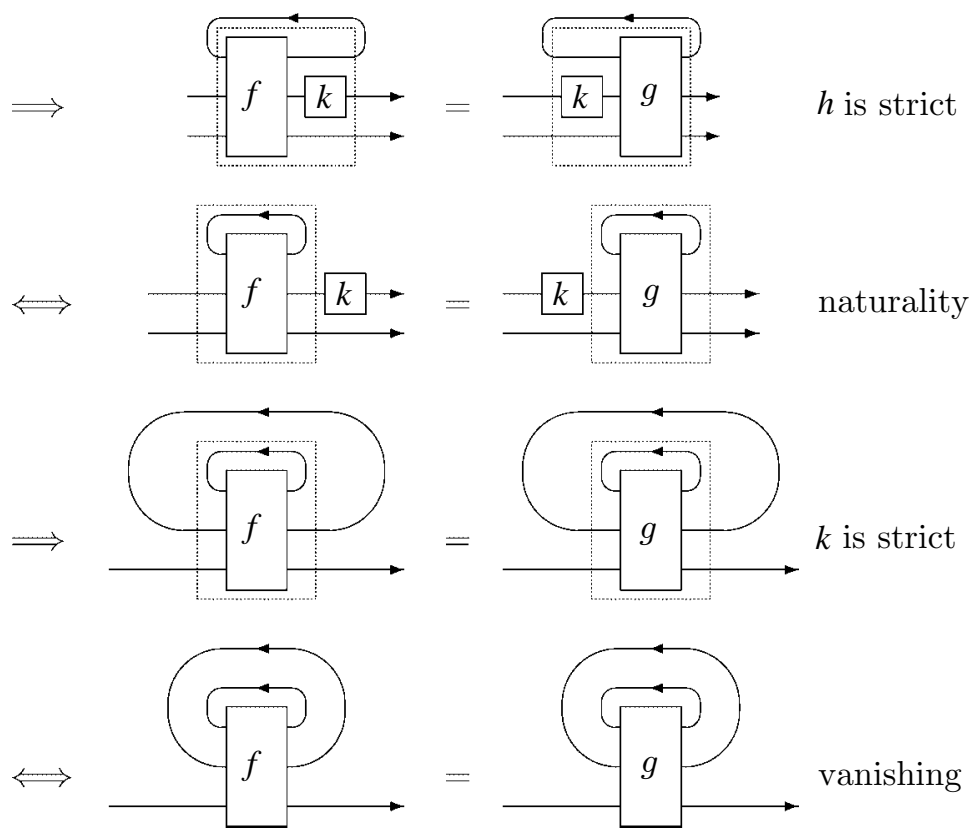

Hence $k \otimes h$ is also strict.

Corollary 4.1. If strict maps are closed under trace, they are also closed under composition (hence form a traced monoidal subcategory).

Proof. Let $h: X \rightarrow Y$ and $k: Y \rightarrow Z$ be strict. The previous lemmas imply that $c_{Y, Z} \circ(h \otimes k): X \otimes Y \rightarrow Z \otimes Y$ is also strict, and we then have $\operatorname{Tr}^{Y}\left(c_{Y, Z} \circ(h \otimes k)\right)=k \circ h: X \rightarrow Z$ strict.

Proposition 4.1. There are traced monoidal categories in which strict maps are not closed under composition.

Proof. Consider the traced monoidal category generated from an object $X$, arrows $\mathrm{f}, \mathrm{g}, \mathrm{h}: X \rightarrow X$ with axioms hohof $=$ gohoh and $\operatorname{Tr}^{X}(k \circ \mathrm{h})=\operatorname{Tr}^{X}(\mathrm{~h})$ for any $k: X \rightarrow X$.

$$
\rightarrow \mathrm{f}-\mathrm{h} \rightarrow \mathrm{h}=\mathrm{h}, \mathrm{g}
$$

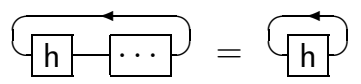

In this traced category, $\mathrm{h}$ is strict but $\mathrm{h} \circ \mathrm{h}$ is not (although $\mathrm{h} \circ \mathrm{h} \circ \mathrm{f}=\mathrm{g} \circ \mathrm{h} \circ \mathrm{h}$, $\left.\operatorname{Tr}^{X}(\mathrm{f}) \neq \operatorname{Tr}^{X}(\mathrm{~g})\right)$. 
Corollary 4.2. $\quad$ There are traced monoidal categories in which strict maps are not closed under trace.

\section{$\S 5 . \quad$ The Trace-Fixpoint Correspondence}

Before going into the main topic of this paper (constructions of traced monoidal categories using uniformity principle), let us recall how traces and fixpoint operators on a category with finite products are related, and see how the uniformity principle on traced monoidal categories generalizes that on categories with fixpoint operator. Later these observations enable us to specialize some of our constructions of traced monoidal categories to those of categories with fixpoint operator.

\section{§5.1. Fixpoint operators}

Let $\mathcal{C}$ be a category with finite products.

Definition 5.1. A parameterized fixpoint operator on $\mathcal{C}$ is a family of functions

$$
(-)^{\dagger}: \mathcal{C}(A \times X, X) \rightarrow \mathcal{C}(A, X)
$$

which is natural in $A$ and satisfies the fixpoint equation $f^{\dagger}=f \circ\left\langle i d_{A}, f^{\dagger}\right\rangle$ : $A \rightarrow X$ for $f: A \times X \rightarrow X$.

Definition 5.2. A Conway operator on $\mathcal{C}$ is a parameterized fixpoint operator $(-)^{\dagger}$ which satisfies

- dinaturality: $\left(f \circ\left\langle\pi_{A, X}, g\right\rangle\right)^{\dagger}=f \circ\left\langle i d_{A},\left(g \circ\left\langle\pi_{A, Y}, f\right\rangle\right)^{\dagger}\right\rangle: A \rightarrow X$ for $f: A \times Y \rightarrow X$ and $g: A \times X \rightarrow Y$

- diagonal property: $\left(f \circ\left(i d_{A} \times \Delta_{X}\right)\right)^{\dagger}=\left(f^{\dagger}\right)^{\dagger}: A \rightarrow X$ for $f: A \times X \times X \rightarrow$ $X$, where $\Delta_{X}: X \rightarrow X \times X$ is the diagonal map.

This axiomatization of Conway operators is taken from [23]; see [3, 9] for other possible axiomatizations.

\section{$\S 5.2$. The correspondence}

The basic relationship between traces and fixpoint operators is

Theorem 5.1 (Hyland/Hasegawa). For any category with finite products, to give a Conway operator is to give a trace (where finite products are taken as the monoidal structure). 
This observation, noticed independently by Martin Hyland and the author, together with some implications to the study on semantics of recursive computation, was first announced in [8]; a full proof is found in [9] (but we should note that mathematically equivalent observations have been made by several authors even before the notion of traced monoidal categories was invented, in particular by Bloom and Ésik [3] and Ştefănescu [24]). Here we shall just recall the constructions of this bijective correspondence:

$$
\begin{gathered}
\frac{f: A \times X \rightarrow X}{f^{\dagger}=\operatorname{Tr}_{A, X}^{X}\left(\Delta_{X} \circ f\right): A \rightarrow X} \\
\frac{g: A \times X \rightarrow B \times X}{\operatorname{Tr}_{A, B}^{X}(g)=\pi_{B, X} \circ\left(g \circ\left(i d_{A} \times \pi_{B, X}^{\prime}\right)\right)^{\dagger}: A \rightarrow B}
\end{gathered}
$$

\section{§5.3. Correspondence of the uniformity principles}

Quite fortunately, the correspondence between traces and Conway operators smoothly extends to the uniformity principles. Let us recall the classical notion of uniformity for fixed points (Plotkin's principle):

Definition 5.3. $\quad$ Let $(-)^{\dagger}$ be a parameterized fixpoint operator on a category with finite products. We say $h: X \rightarrow Y$ is strict (with respect to $(-)^{\dagger}$ ) if the following condition holds:

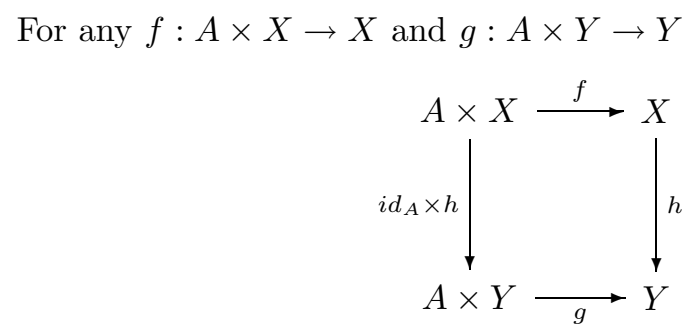

implies $g^{\dagger}=h \circ f^{\dagger}: A \rightarrow Y$.

(The reader should compare this with the corresponding definition for traced monoidal categories (Def. 3.1). They are quite similar - we should confess that Def. 3.1 was inspired from Def. 5.3 - but they are also subtly different, in that the arrow $h$ in the pre-condition of Def. 5.3 survives in the post-condition while it disappears from the post-condition in Def. 3.1.)

For instance, in the category Cppo an arrow is strict with respect to the standard least fixpoint operator if and only if it preserves the bottom, thus is strict in the standard sense. 
It is also possible to formulate the uniformity principle with respect to a given class (quite often a subcategory) $\mathcal{S}$ of strict maps in the same way as Def. 3.2: $(-)^{\dagger}$ satisfies the uniformity principle for the class of arrows $\mathcal{S}$ if, for any $h \in \mathcal{S}$, the condition in Def. 5.3 holds. In [3] (cf. [23]) it is shown that a Conway operator satisfying the uniformity principle for a lluf subcategory with finite products is an iteration operator [3] - thus uniformity principle for a reasonably rich $\mathcal{S}$ does have strong consequences. (But again we shall warn that there are cases where the strict maps do not form a category! Also we note that $\mathcal{S}$ being a category is not necessary to show the above-mentioned result; it suffices to ask that $\mathcal{S}$ contains a few structural morphisms. The only reason to assume $\mathcal{S}$ to be a category seems that it is the case for all natural examples known so far.)

Theorem 5.2. $\quad$ Consider a category with finite products and a Conway operator, and the corresponding trace (as given in Thm. 5.1). Then an arrow is strict w.r.t. the Conway operator if and only if it is strict w.r.t. the trace.

A proof is given in Appendix A. It is almost straightforward to show that the trace-strictness implies the Conway-strictness. The other direction is more non-trivial and slightly tricky; perhaps the easiest way, as taken here, is first to show that Conway-strict arrows are closed under products, using the Bekič property (which gives another axiomatization of Conway operators [8, 9]).

This theorem confirms that the uniformity principles for traces and Conway operators coincide, as long as we talk about those on categories with finite products. We regard this as a strong evidence that our notion of uniformity principle on traces is a natural generalization of that on traditional fixpoint operators in the theory of computation, especially in domain theory. Technically, this result enables us to specialize the constructions of traced monoidal categories via the uniformity principle to those of categories with finite products and Conway operator, to be introduced in the following sections.

\section{$\S 6 . \quad$ Constructions via Uniformity}

Good constructions of categories with trace or fixpoint operator are of great value, as the recent history of knot theory (after 80's we know that many knot invariants can be constructed in a generic way) and domain theory (the progress of axiomatic and synthetic domain theory resulted some constructions of models of domain theory) has shown. The main goal of this paper is a 
small contribution towards this direction: to demonstrate that the uniformity principle on traced monoidal categories helps us with constructing new traced monoidal categories. The construction is of rather general nature, and naturally we cannot expect very strong results. However, we shall try to indicate how natural it is, by pointing the relationship with a classical example: the Scott induction principle in domain theory.

To motivate the constructions, let us start with some elementary exercises:

Let $F, G$ etc be functors between traced monoidal categories which preserve the structure on the nose (which we shall call strict traced functors). Can we give a trace to categories like the comma category $F \downarrow G$, categories of (co)algebras of endofunctors $F$-Alg, F-Coalg, or even the inserters (dialgebras) of $F$ and $G$ etc?

The answer depends on the cases - in general we cannot (as expected), but in some particular cases there exists a natural way to give a trace. It turns out that, for all of these examples, we can naturally identify a full subcategory which is traced monoidal - with help of the uniformity principle.

\section{§6.1. First example: $\mathcal{C}^{-\circ}$}

We shall look at a simple case in detail: given a traced monoidal category $\mathcal{C}$ with a trace $T r$, let us consider the arrow category $\mathcal{C} \rightarrow$, whose objects are arrows of $\mathcal{C}$ and a morphism from $h: X \rightarrow Y$ to $h^{\prime}: X^{\prime} \rightarrow Y^{\prime}$ in $\mathcal{C} \rightarrow$ is a pair $\left(f: X \rightarrow X^{\prime}, g: Y \rightarrow Y^{\prime}\right)$ such that $h^{\prime} \circ f=g \circ h$ holds in $\mathcal{C}$.

$$
(-f \rightarrow,-g \rightarrow):-h \rightarrow \rightarrow-\underline{h} \rightarrow \Longleftrightarrow-f-h^{\prime} \rightarrow=-h-g \rightarrow
$$

$\mathcal{C} \rightarrow$ naturally inherits a symmetric monoidal structure from $\mathcal{C}$, determined by a pointwise manner. The question is then how to give a trace, say, for $(f, g)$ : $k \otimes h \rightarrow l \otimes h$ (i.e., $(l \otimes h) \circ f=g \circ(k \otimes h))$. The natural candidate is the pair $(\operatorname{Tr}(f), \operatorname{Tr}(g))$ - what is not obvious is that if this is really a morphism from $k$ to $l$, i.e., $l \circ \operatorname{Tr}(f)=\operatorname{Tr}(g) \circ k$ holds. At this point, the reader probably notice that we can appeal to the uniformity principle: if $h$ is strict w.r.t. $\mathrm{Tr}$, then $(l \otimes h) \circ f=g \circ(k \otimes h)$ implies $l \circ \operatorname{Tr}(f)=\operatorname{Tr}(g) \circ k !$ Let us define ${ }^{1} \mathcal{C}^{-}$to be a full subcategory of $\mathcal{C} \rightarrow$ whose objects are strict maps w.r.t. the trace. Since strict maps are closed under tensor product, $\mathcal{C}^{-}$is a symmetric monoidal full subcategory of $\mathcal{C} \rightarrow$.

\footnotetext{
${ }^{1}$ The notation " $\mathcal{C}-0$ " is suggested by Martin Hyland.
} 
Proposition 6.1. $\quad \mathcal{C}^{-}$is a traced monoidal category.

Proof. For $(f, g): k \otimes h \rightarrow l \otimes h$ (i.e. $(l \otimes h) \circ f=g \circ(k \otimes h))$, define the trace on $\mathcal{C}^{-}$by $\operatorname{Tr}_{k, l}^{h}(f, g)=(\operatorname{Tr}(f), \operatorname{Tr}(g))$. We have $\operatorname{Tr}_{k, l}^{h}(f, g): k \rightarrow l$ because

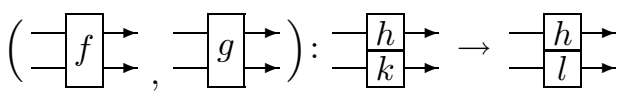

$$
\begin{aligned}
& \Longleftrightarrow \quad-f \stackrel{h}{l} \rightarrow \quad=-\frac{h}{k} \rightarrow g \rightarrow \quad \text { definition } \\
& \Longleftrightarrow \quad f \quad h \quad=-h \stackrel{h}{\longrightarrow}= \\
& \Longrightarrow \quad h \quad h \text { is strict } \\
& \Longleftrightarrow \quad \\
& \Longleftrightarrow(\stackrel{+}{\rightarrow}, \stackrel{\leftrightarrow}{g}):-k \rightarrow \rightarrow-l b \quad \text { definition }
\end{aligned}
$$

The axioms of trace are trivially satisfied.

We shall note that this construction specializes to Conway operators (because the uniformity principles for traces and Conway operators agree): if $\mathcal{C}$ is a category with finite products and a Conway operator, so is $\mathcal{C}^{-}$. Here is a classical example:

Example 6.1 (Scott Induction Principle). Let $D$ be a $\omega$-cpo with bottom $\perp$, and $f: D \rightarrow D$ be continuous. We write fix $(f)$ for the least fixpoint of $f$. Let $P$ be an inclusive (admissible) subset of $D$. If $x \in P$ implies $f(x) \in P$ 
and also $\perp \in P$, then $\operatorname{fix}(f) \in P$ :

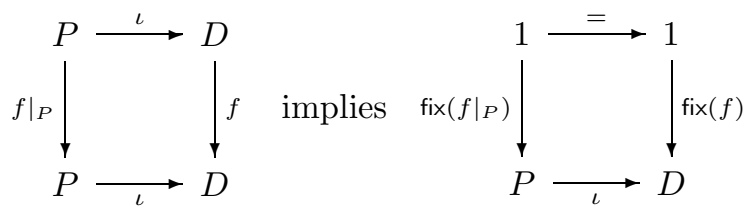

where $\iota$ is the strict order monic associated to the inclusive subset. This can be seen an instance of the construction described above $\left(\mathbf{C p p o}^{-0}\right)$.

This example, although not new at all, gives a strong motivation to our study. It has been observed that many of the proof techniques on type theories like logical relations can be understood as model-construction techniques, for example categorical glueing or comma objects. The example above says that we can understand the Scott induction principle in this general context too, as a construction on traced monoidal categories.

\section{§6.2. Variations}

We have seen that, in a particularly simple case, uniformity principle can be used for constructing new traced monoidal categories from known ones. Now the reader should be able to think of many variations of $\mathcal{C}^{-\circ}$ : like comma categories, categories of algebras of endofunctors, as well as those of coalgebras - just by restricting the objects to be strict with respect to the trace.

Example 6.2 $\left(\mathbf{C p p o}_{\perp}\right.$ from co-slice). $\quad$ It is an easy exercise to see $\mathbf{C p p o}_{\perp}$ as a traced full subcategory of the co-slice $1 \backslash \mathbf{C p p o}$ : its objects are strict maps from the one-point cpo 1 (hence the bottom elements), so arrows are precisely the bottom-preserving maps. The trace on $\mathbf{C p p o}_{\perp}$ is then inherited from Cppo.

Example 6.3 (inserters). Let $F, G: \mathcal{C} \rightarrow \mathcal{D}$ be strict traced functors. We consider the following full subcategory of the inserter of $F$ and $G$ : its objects are pairs $(C, h)$ where $C$ is an object of $\mathcal{C}$ and $h: F C \rightarrow G C$ is a strict map in $\mathcal{D}$, while an arrow $f:(C, h) \rightarrow\left(C^{\prime}, h^{\prime}\right)$ is an arrow $f: C \rightarrow C^{\prime}$ in $\mathcal{C}$ such that $h^{\prime} \circ F f=G f \circ h$ holds. By repeating the same consideration as the case of $\mathcal{C}^{-\circ}$, this forms a traced monoidal category, with the trace inherited from $\mathcal{C}$.

As special cases, we can apply these constructions on categories with finite products and a Conway operator, with strict maps w.r.t. the Conway operator (as we already did in Example 6.1). We already know that the resulting 
category is traced. If its monoidal product is cartesian, by the trace-fixpoint correspondence, we have a Conway operator on it.

\section{$\S 7 . \quad$ Constructions as Enriched Limits}

\section{$\S 7.1$. Some attempts}

Having observed these examples, it is then natural to ask if these constructions can be characterized by some suitable universal property. However, the category of traced monoidal categories and (strict) traced functors fails to have many interesting limits or colimits; even worse, this category is not monadic over Cat (in the sense that it is not monadic for the monad induced by the natural forgetful functor), although it is monadic over the category $\mathbf{S M C a t}{ }_{s}$ of small symmetric monoidal categories and strict symmetric monoidal functors (Martin Hyland and John Power, private communication). Thus this seems not the right setting to look at - in any case our constructions are in much more flavour of two-dimensional limits, and also there seems no way to accommodate the uniformity principle in this one-dimensional view.

Then a second and natural attempt is to look for a suitable enrichment, so that 2-cells somehow capture the strict maps (or natural transformations whose components are strict). As already warned before, strict maps do not form a category in general, so we cannot have a Cat-enrichment. However they do form graphs and it seems natural to consider the following Graph-enrichment on the category of traced monoidal categories and traced functors, for the cartesian closed category Graph $\simeq$ Set $\stackrel{\vec{\rightarrow}}{\rightarrow}$ : each hom-set is equipped with a graph structure whose objects are traced functors, and arrows are monoidal natural transformations whose components are strict.

This seems to work well: for example, it is tempting to characterize $\mathcal{C}^{-}$ as a Graph-cotensor of the graph $(\cdot \rightarrow \cdot)$ and the traced monoidal category $\mathcal{C}$ - here let us recall the notion of cotensors; for general background of enriched category theory see e.g. $[17,4]$.

Definition 7.1. Let $\mathcal{V}$ be a symmetric monoidal closed category and $\mathcal{C}$ be a $\mathcal{V}$-category. We say the cotensor of $V \in \mathcal{V}$ and $C \in \mathcal{C}$ exists if there is an object $[V, C] \in \mathcal{C}$ together with isomorphisms $\mathcal{C}(D,[V, C]) \simeq[V, \mathcal{C}(D, C)]$ which are $\mathcal{V}$-natural in $D$ in $\mathcal{C} . \mathcal{C}$ is $\mathcal{V}$-cotensored when the cotensor of $V$ and $C$ exists for all $V$ and $C$.

(For readers unfamiliar with the notation: note that the square bracket in the right hand side of the defining isomorphism is the internal hom of $\mathcal{V}$; indeed the internal hom can be regarded as the special case of cotensor with $\mathcal{C}=\mathcal{V}$ ). 
Thus we would like to claim

$$
\operatorname{TreMon}\left(\mathcal{B}, \mathcal{C}^{-\circ}\right) \simeq[(\cdot \rightarrow \cdot), \operatorname{TreMon}(\mathcal{B}, \mathcal{C})]
$$

where TreMon is the Graph-enriched category of traced monoidal categories and strict traced functors as described above.

Alas, there already exists a nasty difficulty even in this simple case. The problem is that strict maps in $\mathcal{C}^{-\circ}$ may not agree with those coming from strict maps in $\mathcal{C}$. This is very problematic, as morphisms in $\operatorname{TreMon}\left(\mathcal{B}, \mathcal{C}^{-\circ}\right)$ depend on the strict maps in $\mathcal{C}^{-}$, while those in $[(\cdot \rightarrow \cdot)$, $\operatorname{TreMon}(\mathcal{B}, \mathcal{C})]$ depend just on the strict maps in $\mathcal{C}$.

And, unfortunately, there are counterexamples. Suppose that $\mathcal{C}$ is a traced monoidal category in which all strict maps are monic. Then it follows that $(f, g): h \rightarrow h^{\prime}$ in $\mathcal{C}^{-}$is strict whenever its second component $g$ is strict in $\mathcal{C}$. For instance: the traced monoidal category generated from an object $X$, arrows $\mathrm{f}, \mathrm{g}, \mathrm{h}: X \rightarrow X$ with axioms $\mathrm{h} \circ \mathrm{h} \circ \mathrm{f}=\mathrm{g} \circ \mathrm{h} \circ \mathrm{h}$ and $\operatorname{Tr}^{X}(k \circ \mathrm{h})=\operatorname{Tr}^{X}(\mathrm{~h})$ for any $k: X \rightarrow X$. This has already been used as a case where strict maps do not compose $-\mathrm{h}$ is strict while $\mathrm{h} \circ \mathrm{h}$ is not strict. And in this category every morphism is monic. Therefore $(\mathrm{h} \circ \mathrm{h}, \mathrm{h}): \mathrm{h} \rightarrow i d_{X}$ is strict, although its first component is not.

\section{§7.2. A solution}

Perhaps the easiest way to remedy this is to explicitly specify a "monoidal" subgraph of strict maps (that is, a graph of objects and strict maps which is closed under unit and tensor product) for each traced monoidal category, and then give the enrichment with respect to such explicitly specified graphs of strict maps. For instance, given a traced monoidal category $\mathcal{C}$ with a monoidal subgraph $\mathcal{S}$ of strict maps of $\mathcal{C}$, we define $\mathcal{C}^{-}$as the full subcategory of $\mathcal{C} \rightarrow$ whose objects are in $\mathcal{S}$, and we specify its monoidal subgraph $\mathcal{S}^{-}$as the class of strict maps whose components belong to $\mathcal{S}$.

Now we re-define TreMon as follows. Its objects are traced monoidal categories with a specified monoidal subgraph of strict maps. Arrows are strict traced functors. Its hom-graphs are defined as the previous version, except that we ask the each component of natural transformations stay in the specified monoidal subgraph of strict maps.

Then $\left(\mathcal{C}^{-}, \mathcal{S}^{-}\right)$is indeed the cotensor of the graph $(\cdot \rightarrow \cdot)$ and $(\mathcal{C}, \mathcal{S})$. In fact we have all Graph-cotensors:

Theorem 7.1. TreMon is Graph-cotensored:

$$
\operatorname{TreMon}((\mathcal{B}, \mathcal{U}),[\mathcal{G},(\mathcal{C}, \mathcal{S})]) \simeq[\mathcal{G}, \operatorname{TreMon}((\mathcal{B}, \mathcal{U}),(\mathcal{C}, \mathcal{S}))]
$$


In particular, we have

$$
\operatorname{TreMon}\left((\mathcal{B}, \mathcal{U}),\left(\mathcal{C}^{-}, \mathcal{S}^{-\circ}\right)\right) \simeq[(\cdot \rightarrow \cdot), \operatorname{TreMon}((\mathcal{B}, \mathcal{U}),(\mathcal{C}, \mathcal{S}))]
$$

Explicitly, $[\mathcal{G},(\mathcal{C}, \mathcal{S})]$ can be described as follows. Its objects are graph homomorphisms from $\mathcal{G}$ to $\mathcal{S}$. Arrows are transformations between graph homomorphisms. The symmetric monoidal structure is given by a pointwise manner, e.g. $I(X)=I,(F \otimes G)(X)=F X \otimes G X$ and so on. Given a transformation $\theta: F \otimes H \rightarrow G \otimes H$, we have its trace $\operatorname{Tr}_{F, G}^{H}(\theta): F \rightarrow G$ by $\left(\operatorname{Tr}_{F, G}^{H}(\theta)\right)_{X}=\operatorname{Tr}_{F X, G X}^{H X}\left(\theta_{X}\right)$ (thanks to the uniformity). Finally, we specify the monoidal subgraph part of $[\mathcal{G},(\mathcal{C}, \mathcal{S})]$ as the collection of strict maps whose components are all in $\mathcal{S}$ - in this way we exclude the nasty possibility mentioned before.

We believe that other constructions are naturally characterized as certain Graph-limits in this TreMon, though the details are yet to be spelled out. Also it still remains open how we can characterize the original constructions (without using specified classes of strict maps).

Another direction which might be worth looking at is to replace the graphs by Freyd's paracategories [11, 12] (roughly: categories in which composition is a partial operator), since strict maps form not just a graph but a paracategory, and one may expect "monoidal paracategories" would give a better account on the structure we are looking at.

\section{$\S 8 . \quad$ Strict Maps in $\operatorname{Int} \mathcal{C}$}

The Int construction [16] turns a traced monoidal category $\mathcal{C}$ into a compact closed category [18] called $\operatorname{Int} \mathcal{C}$ to which $\mathcal{C}$ fully faithfully embeds (see Appendix B for a summary of the construction). Its applications to computer science are discussed and studied, especially with the relation to Girard's Geometry of Interaction (GoI) [5] which essentially amounts to implement bidirectional (or interactive) communications via feedback operators, in the literature $[1,13,7]$ - in this context, the Int construction is also known as the GoI construction. It is interesting to see how the uniformity principles in $\mathcal{C}$ and in Int $\mathcal{C}$ are related. However, the situation seems less clear than we first guess, and in this paper we can give only some elementary results and remarks. First, by a straightforward calculation, we have an obvious sort of characterization of strict maps in $\operatorname{Int} \mathcal{C}$ in terms of $\mathcal{C}$ : 
Proposition 8.1. $h \in \operatorname{Int} \mathcal{C}\left(\left(X^{+}, X^{-}\right),\left(Y^{+}, Y^{-}\right)\right)=\mathcal{C}\left(X^{+} \otimes Y^{-}, Y^{+} \otimes\right.$ $\left.X^{-}\right)$is strict in $\operatorname{Int} \mathcal{C}$ if and only if, for any $f \in \mathcal{C}\left(A \otimes X^{+} \otimes X^{-}, B \otimes X^{+} \otimes X^{-}\right)$ and $g \in \mathcal{C}\left(A \otimes Y^{+} \otimes Y^{-}, B \otimes Y^{+} \otimes Y^{-}\right)$,

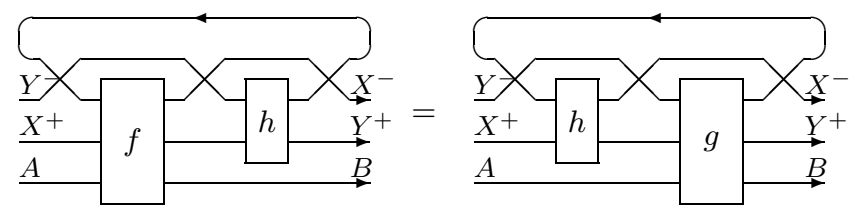

implies

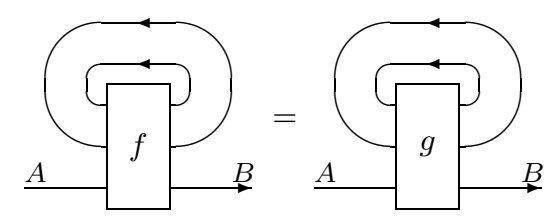

From this characterization, it is immediate to see

Proposition 8.2. If $h^{+} \in \mathcal{C}\left(X^{+}, Y^{+}\right)$and $h^{-} \in \mathcal{C}\left(Y^{-}, X^{-}\right)$are strict in $\mathcal{C}$, then $h^{+} \otimes h^{-} \in \mathcal{C}\left(X^{+} \otimes Y^{-}, Y^{+} \otimes X^{-}\right)=\operatorname{Int} \mathcal{C}\left(\left(X^{+}, X^{-}\right),\left(Y^{+}, Y^{-}\right)\right)$is strict in $\operatorname{Int} \mathcal{C}$.

Therefore the canonical embedding from $\mathcal{C} \times \mathcal{C}^{\text {op }}$ to $\operatorname{Int} \mathcal{C}$ preserves strict maps. However, we do not know much about strict maps in $\operatorname{Int} \mathcal{C}$ which do not arise in this way. We shall conclude this paper with the following simple observations, which indicate the subtlety of this problem.

- The strictness of $h \in \operatorname{Int} \mathcal{C}\left(\left(X^{+}, X^{-}\right),\left(Y^{+}, Y^{-}\right)\right)$in $\operatorname{Int} \mathcal{C}$ does not imply the strictness of $h$ in $\mathcal{C}$ (as a morphism from $X^{+} \otimes Y^{-}$to $Y^{+} \otimes X^{-}$) in general. $^{2}$ As an example, consider the isomorphism $\left(I, X^{*}\right) \stackrel{\simeq}{\rightarrow}(X, I)$ in Int $\mathcal{C}$ where $\mathcal{C}$ itself is a compact closed category. This isomorphism (hence a strict map) amounts to the unit $\eta_{X}: I \rightarrow X \otimes X^{*}$ in $\mathcal{C}$, and its strictness in $\mathcal{C}$ implies $\operatorname{Tr}^{X}\left(i d_{X}\right) \otimes \operatorname{Tr}^{X}\left(i d_{X}\right)=i d_{I}$ which is not always the case (e.g. let $\mathcal{C}$ be the category of finite dimensional vector spaces and linear maps).

- The converse of the above also does not hold in general. For instance, consider the symmetry $c_{I, X} \in \operatorname{Int} \mathcal{C}((I, I),(X, X))=\mathcal{C}(I \otimes X, X \otimes I)$. It is an isomorphism (hence strict) in $\mathcal{C}$, but its strictness in Int $\mathcal{C}$ implies $\operatorname{Tr}^{X}\left(i d_{X}\right) \otimes \operatorname{Tr}^{X}\left(i d_{X}\right)=i d_{I}$.

\footnotetext{
${ }^{2}$ In [10] this was left as an open issue.
} 
(If $\mathcal{C}$ is compact closed, $\operatorname{Int} \mathcal{C}$ is equivalent to $\mathcal{C}$, and the equivalence preserves the strictness: $h \in \operatorname{Int} \mathcal{C}\left(\left(X^{+}, X^{-}\right),\left(Y^{+}, Y^{-}\right)\right)$is strict in $\operatorname{Int} \mathcal{C}$ if and only if the corresponding morphism from $X^{+} \otimes X^{-*}$ to $Y^{+} \otimes Y^{-*}$ is strict in $\mathcal{C}$ - but this characterization does not seem very helpful.)

\section{Appendix A. Proof of Theorem 5.2}

\section{Appendix A.1. From trace-strictness to conway-strictness}

Assume that the diagram

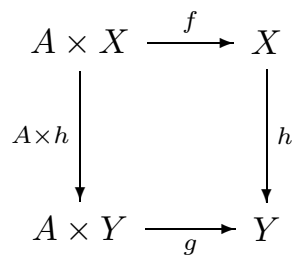

commutes and that $h$ is strict w.r.t. the trace. Then the following diagram

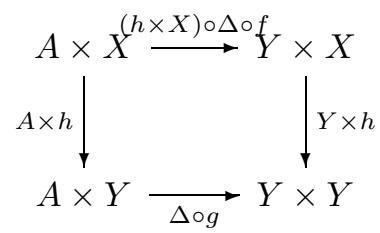

also commutes. From the uniformity for the trace, we have

$$
\operatorname{Tr}^{X}((h \times X) \circ \Delta \circ f)=\operatorname{Tr}^{Y}(\Delta \circ g) .
$$

By Right Tightening, the left hand side is equal to $h \circ \operatorname{Tr}^{X}(\Delta \circ f)$. Since $f^{\dagger}=\operatorname{Tr}^{X}(\Delta \circ f)$ and $g^{\dagger}=\operatorname{Tr}^{Y}(\Delta \circ g)$, we get $h \circ f^{\dagger}=g^{\dagger}$. Therefore $h$ is strict w.r.t. the Conway operator.

\section{Appendix A.2. From conway-strictness to trace-strictness}

Assume that the diagram

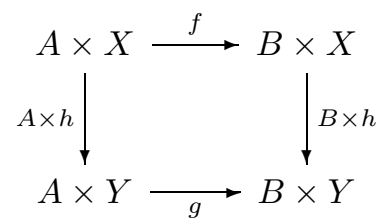


commutes and that $h$ is strict w.r.t. the Conway operator. Then the following diagram

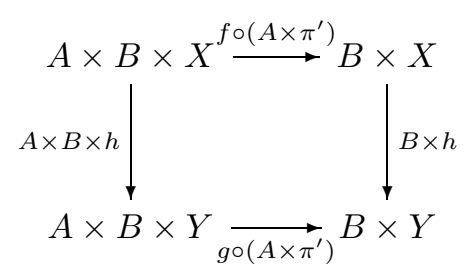

also commutes. Since $h$ is strict w.r.t. the Conway operator, so is $B \times h$, by Lemma 1 below. Thus we have

$$
(B \times h) \circ\left(f \circ\left(A \times \pi^{\prime}\right)\right)^{\dagger}=\left(g \circ\left(A \times \pi^{\prime}\right)\right)^{\dagger} .
$$

Since $\operatorname{Tr}^{X}(f)=\pi \circ\left(f \circ\left(A \times \pi^{\prime}\right)\right)^{\dagger}$ and $\operatorname{Tr}^{Y}(g)=\pi \circ\left(g \circ\left(A \times \pi^{\prime}\right)\right)^{\dagger}$, we get $\operatorname{Tr}^{X}(f)=\operatorname{Tr}^{Y}(g)$. Hence $h$ is strict w.r.t. the trace.

Lemma 1. If $h: X \longrightarrow Y$ and $h^{\prime}: X^{\prime} \longrightarrow Y^{\prime}$ are strict w.r.t. the Conway operator, so is $h \times h^{\prime}: X \times X^{\prime} \longrightarrow Y \times Y^{\prime}$.

Proof. Assume that the diagram

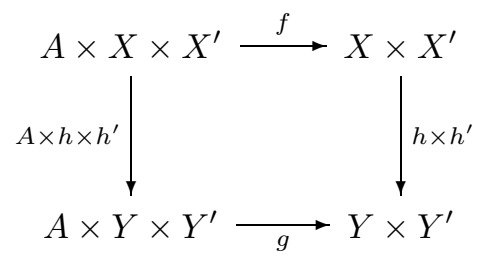

commutes. Our aim is to show $\left(h \times h^{\prime}\right) \circ f^{\dagger}=g^{\dagger}$. By the Bekič property (which holds for any Conway operator $[8,9,23]$ ), this is equivalent to equations

$$
\begin{gathered}
h \circ\left(f_{1} \circ\left\langle A \times X, f_{2}^{\dagger}\right\rangle\right)^{\dagger}=\left(g_{1} \circ\left\langle A \times Y, g_{2}^{\dagger}\right\rangle\right)^{\dagger} \\
h^{\prime} \circ\left(f_{2}^{\prime} \circ\left\langle A \times X^{\prime}, f_{1}^{\prime \dagger}\right\rangle\right)^{\dagger}=\left(g_{2}^{\prime} \circ\left\langle A \times Y^{\prime}, g_{1}^{\prime \dagger}\right\rangle\right)^{\dagger}
\end{gathered}
$$

where $f_{1}=\pi \circ f: A \times X \times X^{\prime} \longrightarrow X, f_{2}=\pi^{\prime} \circ f: A \times X \times X^{\prime} \longrightarrow X^{\prime}$, $f_{i}^{\prime}=f_{i} \circ\left(A \times c_{X^{\prime}, X}\right)$, and so on. We shall show 2. 3 is proved in the same way.

By 1 , the diagrams

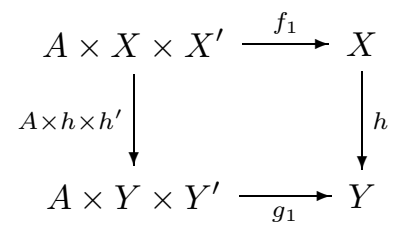




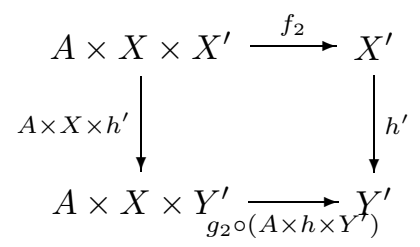

commute. From 5 and the strictness of $h^{\prime}$,

$$
h^{\prime} \circ f_{2}^{\dagger}=\left(g_{2} \circ\left(A \times h \times Y^{\prime}\right)\right)^{\dagger} .
$$

By naturality, the right hand side is equal to $g_{2}^{\dagger} \circ(A \times h)$. Thus we have a commutative diagram

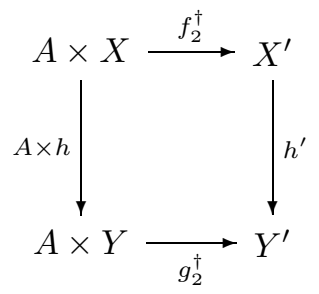

From 4 and 6 ,

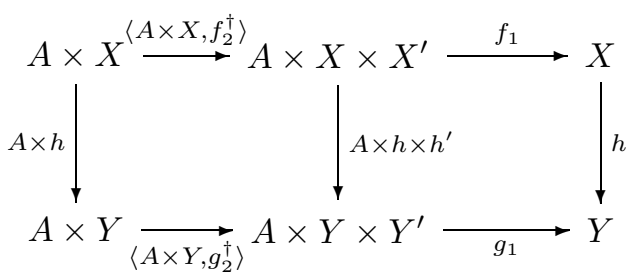

commutes. Since $h$ is strict, we obtain 2 .

\section{Appendix B. The Int Construction}

Let $\mathcal{C}$ be a traced monoidal category. The compact closed category $\operatorname{Int} \mathcal{C}$ is given as follows. Its objects are pairs of those of $\mathcal{C}$, and an arrow from $\left(A^{+}, A^{-}\right)$to $\left(B^{+}, B^{-}\right)$in $\operatorname{Int} \mathcal{C}$ is an arrow from $A^{+} \otimes B^{-}$to $B^{+} \otimes A^{-}$. The identity arrow on $\left(A^{+}, A^{-}\right)$is $i d_{A^{+} \otimes A^{-}} \in \mathcal{C}\left(A^{+} \otimes A^{-}, A^{+} \otimes A^{-}\right)$. The composition of $f \in \operatorname{Int} \mathcal{C}\left(\left(A^{+}, A^{-}\right),\left(B^{+}, B^{-}\right)\right)=\mathcal{C}\left(A^{+} \otimes B^{-}, B^{+} \otimes A^{-}\right)$and $g \in \operatorname{Int} \mathcal{C}\left(\left(B^{+}, B^{-}\right),\left(C^{+}, C^{-}\right)\right)=\mathcal{C}\left(B^{+} \otimes C^{-}, C^{+} \otimes B^{-}\right)$is given by

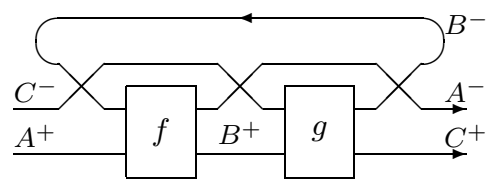


The unit of the monoidal structure is $(I, I)$. The tensor product on objects is $\left(A^{+}, A^{-}\right) \otimes\left(B^{+}, B^{-}\right)=\left(A^{+} \otimes B^{+}, A^{-} \otimes B^{-}\right)$, and on arrows $f:\left(A^{+}, A^{-}\right) \rightarrow$ $\left(B^{+}, B^{-}\right)$and $g:\left(C^{+}, C^{-}\right) \rightarrow\left(D^{+}, D^{-}\right)$we have

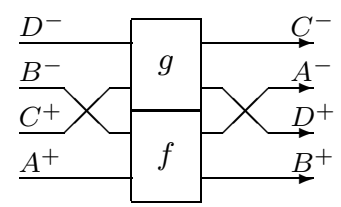

The symmetry from $\left(A^{+}, A^{-}\right) \otimes\left(B^{+}, B^{-}\right)$to $\left(B^{+}, B^{-}\right) \otimes\left(A^{+}, A^{-}\right)$in $\operatorname{Int} \mathcal{C}$ is $c_{A^{+}, B^{+}} \otimes c_{A^{-}, B^{-}}$in $\mathcal{C}$. The duality $(-)^{*}:(\operatorname{Int} \mathcal{C})^{\text {op }} \rightarrow \operatorname{Int} \mathcal{C}$ is given by $\left(A^{+}, A^{-}\right)^{*}=\left(A^{-}, A^{+}\right)$and $f^{*}=c_{B^{+}, A^{-}} \circ f \circ c_{B^{-}, A^{+}}$for $f:\left(A^{+}, A^{-}\right) \rightarrow$ $\left(B^{+}, B^{-}\right)$. The unit and counit

$$
\begin{aligned}
& \eta_{\left(A^{+}, A^{-}\right)}:(I, I) \rightarrow\left(A^{+}, A^{-}\right) \otimes\left(A^{+}, A^{-}\right)^{*}=\left(A^{+} \otimes A^{-}, A^{-} \otimes A^{+}\right) \\
& \varepsilon_{\left(A^{+}, A^{-}\right)}:\left(A^{+}, A^{-}\right)^{*} \otimes\left(A^{+}, A^{-}\right)=\left(A^{-} \otimes A^{+}, A^{+} \otimes A^{-}\right) \rightarrow(I, I)
\end{aligned}
$$

are given by the suitable isomorphisms in $\mathcal{C}$.

Like any compact closed category ${ }^{3}, \operatorname{Int} \mathcal{C}$ has a unique trace, called canonical trace [16]. To be explicit, for $f:\left(A^{+}, A^{-}\right) \otimes\left(X^{+}, X^{-}\right) \rightarrow\left(B^{+}, B^{-}\right) \otimes$ $\left(X^{+}, X^{-}\right)$, its trace $\operatorname{Tr}^{\left(X^{+}, X^{-}\right)}(f):\left(A^{+}, A^{-}\right) \rightarrow\left(B^{+}, B^{-}\right)$is given as follows.

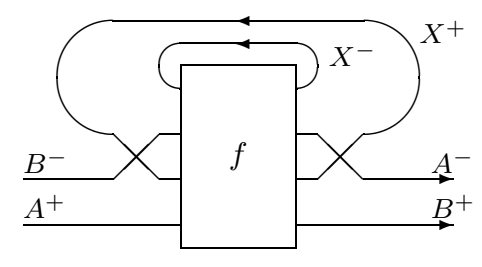

\section{Acknowledgements}

I thank John Power and Peter Selinger for helpful suggestions, and Gheorghe Ştefănescu for pointers to related work.

\section{References}

[1] Abramsky, S., Retracing some paths in process algebra, Proc. Concurrency Theory (CONCUR'96), Springer Lecture Notes in Comput. Sci., 1119 (1996), 1-17.

\footnotetext{
${ }^{3}$ It seems to be folklore that any compact closed category (in fact any tortile category) has a unique trace.
} 
[2] Arbib, A. M. and Manes, E. G., Partially additive categories and flow diagram semantics, J. Algebra, 62 (1980), 203-227.

[3] Bloom, S. and Ésik, Z., Iteration Theories, EATCS Monographs on Theoretical Computer Science, Springer-Verlag, 1993.

[4] Borceux, F., Handbook of Categorical Algebra 2: Categories and Structures, Encyclopedia Math., 51, Cambridge University Press, 1994.

[5] Girard, J.-Y., Geometry of interaction I: interpretation of system F, Proc. Logic Colloquium (1989), pp. 221-260.

[6] Gunter, C. Semantics of Programming Languages, MIT Press, 1992.

[7] Haghverdi, E., A Categorical Approach to Linear Logic, Geometry of Interaction and Full Completeness, $\mathrm{PhD}$ thesis, University of Ottawa, 2000.

[8] Hasegawa, M., Recursion from cyclic sharing: traced monoidal categories and models of cyclic lambda calculi, Proc. Typed Lambda Calculi and Applications (TLCA'97), Springer Lecture Notes in Comput. Sci., 1210 (1997), pp. 196-213.

[9] Models of Sharing Graphs: A Categorical Semantics of let and letrec, PhD thesis ECS-LFCS-97-360, University of Edinburgh, 1997/Distinguished Dissertation Series, Springer-Verlag, 1999.

[10] - The uniformity principle on traced monoidal categories, Proc. Category Theory and Computer Science (CTCS'02), Electron. Notes Theor. Comput. Sci., 69 (2003).

[11] Hermida, C. and Mateus, P., Paracategories I: internal paracategories and saturated partial algebras, Theoret. Comp. Sci., 309 (2003), 125-156.

[12] - Paracategories II: adjunctions, fibrations and examples from probabilistic automata theory, Theoret. Comp. Sci., 311 (2004), 71-103.

[13] Hildebrandt, T. T., Panangaden, P. and Winskel, G., A relational model of nondeterministic dataflow, Proc. Concurrency Theory (CONCUR'98), Springer Lecture Notes in Comput. Sci., 1466 (1998), 613-628.

[14] Joyal, A. and Street, R., Geometry of tensor calculus I, Adv. Math., 88 (1991), 55-113.

[15] Braided tensor categories, Adv. Math., 102 (1993), 20-78.

[16] Joyal, A., Street, R. and Verity, D., Traced monoidal categories, Math. Proc. Cambridge Phil. Soc., 119 (1996), 447-468.

[17] Kelly, G. M., Basic Concepts of Enriched Category Theory, London Math. Soc. Lecture Note Ser., 64, Cambridge University Press, 1982.

[18] Kelly, G. M. and Laplaza, M. L., Coherence for compact closed categories, J. Pure Appl. Algebra, 19 (1980), 193-213.

[19] Mac Lane, S., Categories for the Working Mathematician, Grad. Texts in Math. 5, Springer-Verlag, 1971.

[20] Plotkin. G. D., Domains, The "Pisa" Notes, 1983.

[21] Selinger, P., Categorical structure of asynchronomy, Proc. 15th Mathematical Foundations of Programming Semantics (MFPS), Electron. Notes Theor. Comput. Sci., 20 (1999).

[22] Shum, M. C., Tortile tensor categories, J. Pure Appl. Algebra, 93 (1994), 57-110.

[23] Simpson, A. and Plotkin, G., Complete axioms for categorical fixed-point operators, Proc. 15th Logic in Computer Science (LICS 2000), pp. 30-41.

[24] Ştefănescu, G., Network Algebra, Discrete Mathematics and Theoretical Computer Science Series, Springer-Verlag, 2000.

[25] Yetter, D. N., Functorial Knot Theory, World Scientific, 2001. 